\title{
THE INFLUENCE OF 54970) EDTMP-CONCENTRATION ON THE BIODISTRIBUTION OF RADIO-LANTHANIDES AND 225-Ac IN TUMOR BEARING MICE
}

\author{
G.J.BEYER \\ Division of Nuclear Medicine, Cantonal Hospital of the University of Geneva \\ R.OFFORD, G.KÜNZI, Yu.ALEKSANDROVA, U.RAVN \\ Department of Medical Biochemistry, University of Geneva \\ S.JAHN, J.BARKER,O.TENGBLAD, M.LINDROOS, \\ and the ISOLDE Collaboration \\ CERN, PPE Division, 1211-Geneva 23, Switzerland
}

\begin{abstract}
SUMMARY
High resolution gamma spectroscopy was applied to measure simultaneously the biodistribution of carrier-free radionuclides of several lanthanides ( ${ }^{141} \mathrm{Ce}, 145 \mathrm{Sm},{ }^{149} \mathrm{Gd}$, $167 \mathrm{Tm})$ and $225 \mathrm{Ac}$ in tumor bearing nude mice. Mixtures of the radio-tracers were injected in solutions containing different concentrations of EDTMP (EDTMP = ethylenediaminetetramethylenephosphonic acid). The strong dependence of liver uptake on the ionic radius of the radio-lanthanides was confirmed for all tracers used. The ratios of radioactivity concentrated in tumor to that concentrated in liver are strongly influenced by the EDTMP concentration, reaching values close to 10 for $\mathrm{Tm}, 3$ for $\mathrm{Sm}$ and 1 for Ac. The optimal EDTMP concentrations, giving highest tumor to liver ratios of enrichment, were between 1 and $10 \mathrm{mM}$ for $100 \mu \mathrm{l}$ injected volume for the animal model used in this experiment. In radionuclide therapy using EDTMP as ligands close control of ligand concentration will be necessary.
\end{abstract}

CERN LIBRARIES, GENEVA

( IS 330 and IS 331 )

Subitted to Nuclear Medicine and Biology 1996 


\section{INTRODUCTION}

It is now well established that radio-lanthanides are nonspecific "tumor seeking" tracers. The heavy lanthanides and yttrium are most promising because of relatively low liver uptake $(13,2,3,8,9)$ The rare earth elements show a significantly faster blood clearance in comparison to $\mathrm{Ga}(9$ ), probably due to the fact that they do not form stable complexes with transferrine $(17,20)$. The main pathways for the biodistribution of carrier-free rare earth elements end in renal excretion, bone and liver accumulation. When injected as citrate solution the light lanthanides and Ac show a very high liver uptake (more than $50 \%$ in case of mice or rats) but the bone uptake is moderate (6). On the other hand the heavy lanthanides including yttrium show acceptably low liver uptake but high bone accumulation, and renal excretion is also significantly higher compared to the light rare earth elements $(6,7)$. The tumor uptake does not significantly change within the lanthanides as long as carrier-free radiotracers are used. It has been concluded that chelating ligands of low molecular weight could help to reduce the hydrolysis of lanthanide species in the liver and consequently help to improve the tumor to liver ratios, and indeed, chelating ligands like $\alpha$-HIB ( $\alpha$-hydroxyisobutyric acid) or NTA (nitrilitriacetic acid) showed promising results $(18,19)$. However, when injected in combination with chelating ligands forming very stable complexes the rare earth elements are rapidly excreted via the kidney and are consequently lost. Since the chelating ligands and consequently the stability constants of the complexed ions formed play a such important role for the biological behavior the overall concentration of the low molecular weight chelators must have a significant influence as well. In the case of citrate we could see such an concentration effect only at hypertonic concentrations not applicable in practice (4).

In the present study we have chosen EDTMP as the ligand. It is assumed that, as a phosphonic acid containing ligand EDTMP would favour bone uptake of $\operatorname{Sm}(12)$. On the other hand it has been clearly demonstrated that EDTMP reduces significantly the liver uptake of radiolanthanides $(5,12,16)$. The aim of our study is, rather than further collection of data on radio-tracer accumulation expressed in \%/g tissue, to evaluate relationships between physico-chemical parameters of the injected compound (ionic radius of the radionuclide, complex formation stability) and bio-distribution. Such information is required to contribute to our currently incomplete understanding on mechanisms for the tumor accumulation of radio-lanthanides, and could help to design more efficient radiopharmaceuticals for diagnosis and therapy. Rare earth elements are most suitable for such studies since they show a fine graduation of chemical properties from one element to the next. In contrast to most radioisotope work, one can move from one rare earth element to the next without having to change the chelator, a great advantage for optimization studies. In addition to the rare earth elements $225 \mathrm{Ac}$ was taken into the experimental series because of its special therapeutical interest ( 11 ). 


\section{EXPERIMENTAL}

\section{Radionuclides:}

Highest quality radio-tracers are required: they must be truly carrier-free and isotopically clean. The different radionuclides used in this experiment were produced at the on-line isotope separator facility ISOLDE at CERN ( 14 ). The $1 \mathrm{GeV}$ proton beam of the CERN booster interacts with the thick (50 - 200 $\mathrm{g} / \mathrm{cm}^{2}$ ) ISOLDE target which is kept at very high temperatures $\left(\geq 2000^{\circ} \mathrm{C}\right)$. The nuclear reaction products formed in spallation, fission or fragmentation are ionized in an ion source and extracted as single positively charged ions by $60 \mathrm{kV}$. By passing through the magnet they are separated according to their mass to charge ratio. Isotopically pure radionuclides can be extracted and collected. The radio-lanthanides ${ }^{167} \mathrm{Tm},{ }^{153} \mathrm{Gd},{ }^{149} \mathrm{Gd},{ }^{147} \mathrm{Eu}$ and ${ }^{145} \mathrm{Sm}$ were produced from a Ta-foil target, and ${ }^{225} \mathrm{Ac},{ }^{141} \mathrm{Ce},{ }^{147} \mathrm{Nd}$ and ${ }^{153} \mathrm{Sm}$ from a U-carbide target. For the final radiochemical purification (separation of isobars) ion exchange chromatography (system Aminex $\mathrm{A} 5 / \alpha-\mathrm{HIB}, \mathrm{pH}=5.0$ ) was applied. The final preparations of the radiotracers were of high concentration $(>50 \mathrm{MBq} / \mathrm{ml}$ ) in $0.05 \mathrm{M} \mathrm{HCl}$ and carrier-free.

\section{EDTMP:}

The EDTMP-ligand was synthesized according to the method described by Moedritzer ( 15 ) and purified according Garlich ( 10 ).

\section{Animals:}

In total 30 nude mice bearing xenografted tumors were used in this study. The tumor used was a human colon carcinoma T380. It was transplanted subcutaneously into female Swiss nu/nu mice from Iffa Credo, L'Arbresle, France. The animal experiments have been carried out under the authorization number 31.1.1003/794/II.

\section{Injection solution:}

For each animal $100 \mu \mathrm{l}$ of an injection solution was prepared separately by mixing appropriate volumes of stock solutions. The EDTMP concentration was varied over several orders of magnitude up to $30 \mathrm{mM}$. The "blank" solution (radionuclides with no EDTMP) contained citrate at a concentration of $1 \mathrm{mM}$ to avoid radio-colloid formation. The radionuclide mixture contained about $50 \mathrm{kBq}$ of each isotope for one animal. The $\mathrm{pH}$ of the injection solution was 6.5. High-tension paper electrophoresis (Whatman $3 \mathrm{~mm} /$ pyr./HAc/water 2.5:1:225, $\mathrm{pH}=6.5$ ) showed that the radionuclides move as negatively charged ions in presence of EDTMP (10 mM) into the direction of the anode. No further peaks could be detected.

\section{Procedure:}

The individually prepared $100 \mu 1$ injection solutions were injected into the tail vein of the tumor bearing nude mice. The mice were killed $15 \mathrm{~h}$ after injection. Urine and faeces were carefully combined and the 
main organs were collected. The radioactivity present due to each individual isotope was determined by using a high resolution Ge-spectrometer. The gamma-spectroscopic measurement was performed after establishing the radioactive equilibrium conditions required in case of the $225 \mathrm{Ac}$. The measuring time of the samples depended greatly on the amount of activity present. Samples containing of the order of $\mathrm{kBq}$ of the radiotracers were measured between 300 and 1000 seconds, while weak samples like blood or fat were often measured overnight in order to obtain information even at radioactivity levels as low as 100 $\mathrm{mBq}$ per sample. Several series of experiments have been performed: in one study the EDTMPconcentration was varied between 0.01 and $10 \mathrm{mM}, 225 \mathrm{Ac},{ }^{141 \mathrm{Ce}},{ }^{149} \mathrm{Gd}$ and $167 \mathrm{Tm}$ was used as radiotracers, and in a second the same tracers were used to extend the EDTMP-concentration range to 30 $\mathrm{mM}$. In a third study a mixture of ${ }^{145} \mathrm{Sm}, 153 \mathrm{Gd}$ and $225 \mathrm{Ac}$ was used as radio-tracers.

\section{RESULTS AND DISCUSSION}

In total, more than 300 gamma spectra have been recorded and analyzed in terms of the content of the different radioisotopes used. The experimental results are presented in Fig.1-4. We discuss here the experimental results in terms of the influence of ligand concentration on the specific radionuclide uptake in different tissues; the influence of ionic radius on the specific uptake; and the ratios of enrichment as function of EDTMP concentration and the ionic radii of the radionuclides.

\section{Influence of the EDTMP concentration on the specific organ uptake (Fig.1, Tab.1)}

BLOOD: $\quad$ From earlier studies we learned that when low molecular weight chelators are used radiolanthanides are cleared fast from blood, and usually no significant change in the biodistribution was found between several hours and one day $(5,6,8,9)$. In the present study the low radioactivity concentrations found in blood $(<0.1 \% / g$ in all cases) confirm these earlier findings. Comparing the different elements it can be seen, that Tm shows the fastest clearance. With growing EDTMP concentration the clearance for all elements used became increased. The detection limit for the experimental conditions used was as low as 0.001 to $0.002 \% / \mathrm{g}$ depending on the radionuclide, due to the long measuring times.

MUSCLE: The uptake in muscle is generally very low (around $0.1 \% / g$ ). The detection limit was about $0.01 \% / \mathrm{g}$. The uptake of Ac and Ce is slightly higher compared to Gd and Tm. At EDTMP concentrations $>0.05 \mathrm{mM}$ a slight progressive decrease of radionuclide uptake in muscles can be seen.

FAT: $\quad$ The radionuclide uptake in fat tissue was generally higher compared to muscles but the trend was identical: Ac and Ce showed the higher uptake in comparison with Gd and Tm and the uptake was reduced at higher EDTMP concentrations $(>0.1 \mathrm{mM})$. The detection limit was of the order of $0.2 \% / g$ because of small sample weights. 
HEART: $\quad$ The specific radionuclide uptake in myocardial tissue is surprisingly high. The Ac uptake is more than one order of magnitude higher than those of Tm. Increased EDTMP concentration reduces the uptake significantly. Detection limit was of the order of $0.2 \% / g$ because of small sample weights.

LIVER: $\quad$ The liver uptake is dramatically changed with EDTMP concentration. When EDTMP is absent $([\mathrm{EDTMP}]=0,[$ citrate $]=1 \mathrm{mM})$ or at very low EDTMP concentrations, Ac and $\mathrm{Ce}$, the elements with the largest ionic radius, showed a very high liver uptake (around $50 \%$ ). This was expected and confirmed earlier results. On the other hand, the uptake of Tm under the same conditions is by one order of magnitude smaller. The liver uptake of all radiotracers used in this experiment decreased proportionally, over almost two orders of magnitude, with increasing EDTMP concentration. The liver uptake of Ac was the highest among the radionuclides used, but could be brought down by $20 \mathrm{mM}$ EDTMP to approximately the level of radionuclide uptake seen with citrate (without EDTMP) for Tm, the element having the lowest uptake. The values for $\mathrm{Gd}, \mathrm{Sm}$ and $\mathrm{Ce}$ are in-between those of $\mathrm{Tm}$ and $\mathrm{Ac}$, according to their ionic radius as shown in Fig. 2 . The detection limit was about $0.2 \% / g$ due to short measuring times.

SPLEEN: $\quad$ The specific uptake in the spleen is approximately an order of magnitude less than that in the liver. But in principle the same general picture was obtained: a steady decrease of uptake with increased EDTMP concentration. The detection limit was $0.1 \% / g$ due to small sample weights and short measuring times.

URINE: $\quad$ The experimental date are given in $\%$ of injected dose in this case. The combined urinary and fecal excretions are discussed as urinary excretion only, an assumption which has been proved in earlier experiments and is realistic because the very low mobility of liver bound radiolanthanides limits their subsequent excretion. It is clearly seen that at low EDTMP concentrations $(<0.01 \mathrm{mM})$ practically no Ac is excreted, but for $\mathrm{Tm}$ the expected $20 \%$ excretion was measured for [EDTMP] $=0$. The excretion rate of the other rare earth elements are in between. At high EDTMP concentrations similar excretion rates were found for all lanthanides: the value for $\mathrm{Ac}$, however, is still a little smaller than the others even at the highest EDTMP concentration used in this experiment. The relative errors of this measurement is of the order of $15 \%$ due to geometrical sample errors.

KIDNEY: $\quad$ EDTMP reduced the kidney uptake generally by one order of magnitude. For the Ac and Ce uptake the influence of EDTMP can be seen only at higher EDTMP concentrations ( $>0.01 \mathrm{mM})$. In case of Tm, however, very low EDTMP concentrations already reduce the kidney uptake significantly. FEMUR: $\quad$ Practically all measured values for bone uptake are within 15 and $40 \% / \mathrm{g}$. No significant influence of the EDTMP concentration can be evaluated from the data. Only at high EDTMP concentrations ( $>10 \mathrm{mM}$ ) a decrease of radionuclide uptake was observed, however, identically for all radiotracers used. 
TUMOR: $\quad$ Strikingly a very similar behavior to bone is observed for the tumor uptake. The differences between the uptake behavior of the different lanthanides are hardly significant. The influence of EDTMP concentration is weak, except at concentrations $>10 \mathrm{mM}$. Interestingly, in all cases Ac showed the highest tumor uptake. The detection limit was of the order of $0.05 \% / g$.

\section{Influence of the ionic radius on the specific radionuclide uptake in different tissue}

Fig. 2 shows the radionuclide uptake for the main pathways as a function of the ionic radius of the radioisotopes: an EDTMP concentration of $10 \mathrm{mM}$ was chosen for this example. Only the liver uptake showed a significant relationship to the ionic radius under this condition: the liver uptake changes by a factor of 40 comparing the uptake of Tm and Ac while the tumor uptake changes only by $50 \%$. We believe that the most important finding is the significantly higher specific tumor uptake of $\mathrm{Tm}$, seen as a representative of the heavy rare earth region, compared to liver uptake. No influence of the ionic radius in the bone (femur) uptake can be observed. The urinary excretion is more or less identical for all lanthanides. As mentioned above the Ac excretion is still by about $20 \%$ smaller at [EDTMP] $=10 \mathrm{mM}$.

\section{Ratios of enrichment ( Tab.2)}

The maximum value for the tumor to blood ratio was measured to be 300 , for tumor to muscle around 100 , and for tumor to liver around 10. The tumor to organ ratios generally increase with the EDTMP concentration in case of blood, muscle, fat, heart, spleen, and liver, but remain more or less constant in case of femur and kidney. As the most important result we illustrate in Fig. 3 the tumor to liver ratios of radionuclide uptake versus the EDTMP concentration. With growing EDTMP concentration the tumor to liver ratios clearly increase reaching maximum values in the region of 1 - 10 mM EDTMP. With this finding we confirm results of earlier studies $(4,5)$. The maximum values for the tumor to liver ratios are around 10 for $\mathrm{Tm}, 6$ for Gd, 2 for Sm and below 1 for $\mathrm{Ce}$ and Ac. The tumor to femur ratios remain, however, more or less constant. In Fig.4, tumor to organ ratios are plotted versus the ionic radii for an EDTMP concentration of $10 \mathrm{mM}$. The tumor to liver ratio is changed by nearly two orders of magnitude, while a change by a factor of about 2 only can be seen for most of the other tissues. The tumor to femur ratios, however, remain more or less constant. 


\section{CONCLUSION}

EDTMP is a suitable ligand for using radionuclides of all rare earth elements and Ac as bone and tumor seeking tracers. EDTMP changes significantly the bio-distribution of radio-lanthanides and Ac but it does not lead to higher bone uptake compared to the citrates of the same elements. The tumor uptake is not higher as well, however, the great advantage of the EDTMP ligand is the fact that, depending on the ligand concentration, the liver uptake of all rare earth elements and Ac can be reduced by nearly 2 orders of magnitude compared to the citrates. On the other hand the tumor uptake is significantly reduced at high EDTMP concentration (> $10 \mathrm{mM}$ ) only, and consequently the tumor to liver ratios are improved by about one order of magnitude for all lanthanides and Ac. The mechanism of the prevention of liver uptake of lanthanides by EDTMP is not yet fully understood, but it is very likely to depend at least in part on the stability of chelate complex formation. The same behaviour as above was observed earlier for radioyttrium ( 5 ).

The biodistribution of $225 \mathrm{Ac}$ injected in $20 \mathrm{mM}$ EDTMP solution is practically identical with that of ${ }^{167} \mathrm{Tm}$-citrate and presumably, since $\mathrm{Tm}$ and $\mathrm{Y}$ injected as citrates show nearly identical biokinetic behaviour ( 7 ), also of $90 \mathrm{Y}$-citrate. Consequently, one can expect a greater therapeutic efficacy in endoradionuclide therapy of bone metastases when replacing $90 \mathrm{Y}$-citrate by ${ }^{225} \mathrm{Ac}$-EDTMP due to the $\alpha$ radiation.

Furthermore, the systematic change of the bio-distribution along the lanthanides injected in EDTMP solutions allows the finding of optimal conditions for any radioisotope of these elements. Most interesting in this area is ${ }^{149} \mathrm{~Tb}$ which, partially decaying by alpha emission, could be very suitable for radionuclide therapy as an EDTMP complex, as well as in combination with monoclonal antibodies, as proposed by Allen (1).

\section{Acknowledgement}

The authors wish to acknowledge the CERN Directory generally and the PPE Directory in particular for their continous interest in our bio-medical oriented work and for providing the excellent experimental conditions. The thanks goes also to the staff of the PS Booster for their efforts to provide the proton beam in the required high quality needed for our experiments, to our colleagues from ISOLDE for the continuos assistance and help during the collections and finally to the radio protection group for their excellent assistance in organising the fast shipments of the preparations. 


\section{REFERENCES}

1. Allen B.J., Blagojevic N., Meriaty H., Leigh J. (1993)

Role of alpha and beta emitting radiolabelled monoclonal antibodies in therapy of subclinical metastases

Clinical Oncological Society Australia, Perth, 154

2. $\quad$ Ando A., I. Ando I, Hiraki T., Takeshita M. and Hisada K. (1982)

Mechanism of tumor and liver concentration of ${ }^{111} \mathrm{In}$ and ${ }^{169} \mathrm{Yb}$ : ${ }^{111} \mathrm{In}$ and ${ }^{169} \mathrm{Yb}$ binding substances in tumor tissues and liver

Eur. J. Nucl. Med. 7, 298-303

3. Ando A., Ando I, Sakamoto K., Hiraki T., Hisada K. and Takeshita M. (1983)

Affinity of ${ }^{167} \mathrm{Tm}$-citrate for tumor and liver tissue

Eur. J. Nucl. Med. 8, 440-446

4. Beyer G.J., Franke W.G., Hennig K, Johannsen B.A., Khalkin V.A., Kretzschmar M., Lebedev N.A., Münze R., Novgorodov A.F. and Thieme K. (1978)

Comparative kinetic studies of simultaneously injected ${ }^{167} \mathrm{Tm}$ - and ${ }^{67} \mathrm{Ga}$-citrate in normal and tumour bearing mice

Int. J. Appl. Radiat. Isotopes 29,673-680

5. Beyer G.J., Münze R., Fromm W.D., .Franke W.G., Henke E., Khalkin V.A., Lebedev N.A. (1981)

Spallation produced ${ }^{167} \mathrm{Tm}$ for medical application

"Radionuclide Imaging 1980" IAEA Vienna, Vol.1 pp.587 (IAEA-SM-247/60) 1981

6. Beyer G.J., Schomäcker K., BergmannR. and Schäfer G. (1989)

Simultaneous measurement of the ${ }^{87} \mathrm{Y}$ - and ${ }^{167} \mathrm{Tm}$-biokinetics in rats

ZfK - 669, Annual Report 1988, edited by R.Münze, Rossendorf, May 1989, p. 98

7. Beyer G.J., Bergmann R., Schomäcker K., Rösch F., Schäfer G., Kulikov E.V., Novgorodov A.F. (1990)

Comparison of the Biodistribution of ${ }^{225} \mathrm{Ac}$ and Radio-lanthanides as Citrate Complexes

Isotopenpraxis 26 (1990) 111-114

8. Beyer G.J., Bergmann R., Kampf G., Mäding, P., Rösch F. (1992)

Simultaneous study of the biodistribution of radio-yttrium complexed with EDTMP and CITRATE ligands in tumour bearing rats

Nuclear Medicine and Biology, Int.J.of Radiat.Appl.and Instr. Part B 19, 201

9. Beyer G.J., Kampf G., Bergmann R. (1995)

Influence of ligand concentration on the biodistribution of ${ }^{87} \mathrm{Y}$ and ${ }^{67} \mathrm{Ga}$ in tumor bearing rats

Eur.J.Nucl.Med. 22 (8), 914 (ENMR 757)

10. Garlich Joseph R., Simon J. and Masterson T.T. (1990)

Method of purifying aminomethylenephosphonic acids for pharmaceutical use United States Patent, Patent Nr: 4,937,333 Date of patent: June 26, 1990

11. Gerling M.W., Kaspersen F.M., Apostolidis C., van der Hout R. (1993)

The feasibility of $225 \mathrm{Ac}$ as a source of alpha-particles in radioimuno therapy

Nucl.Med.Commun. 14, 121-125

12. Goeckeler W.E., Edwards B., Volkert W.A., Holmes R.A., Simon J., Wilson D. (1987)

Skeletal localisation of ${ }^{153} \mathrm{Sm}$ chelates: potential therapeutic bone agents

J.Nucl.Med. 28, (4) 495-504 
13. Hisada K., Ando A., Hirakati T. and Ando I. (1971)

Recent development of tumor affine radioisotopes

Sogo-Tinsho 20, 1772-1783

14. Kugler E., Fiander D., Jonson B., Haas H., Przewloka A., Ravn H.L., SimonD., Zimmer K. (1992)

The ISOLDE facility at the CERN PS booster

Nucl.Instr.Meth. B70, 41

15. Moedritzer K and Irani R.R. (1966)

The direct synthesis of a -amino-methyl-phosphonic acids, Mannich type reaction with orthophosphorous acid

J.Org.Chem. 34, 1603

16. Rösch F., Neumaier B., Stöcklin G., Qaim S.M., Herzog H., Müller-Gärtner W.H. (1993)

${ }^{86} \mathrm{Y}$ citrate and ${ }^{86} \mathrm{Y}$ DETMP: Preparation, chemical characterisation and in-vivo quantitation of their pharmacological data with PET

10-th International Symposium on Radiopharmaceutical Chemistry, Kyoto, pp.546-548

17. Schomäcker, K, W.G.Franke, A.Gottschalk, C.Hartwig, A.Richter, G.J.Beyer (1986)

Physicochemical properties and biokinetics of tumor-affine metal(M)-ligand complexes.

In: Nuclear Medicine in Clinical Oncology (Ed. C. Winkler), Springer Verlag, Berlin, Heidelberg, pp. 397-401

18. Schomäcker K., W.G.Franke, G.J.Beyer, R.Münze (1986)

Radiopharmacology of tumor-seeking trivalent metal complexes.

In: Radioaktive Isotope in Klinik und Forschung, Hsg.: R.Höfer und H. Bergmann,

Verlag H.Egermann, Wien, pp. 97-102

19. Schomäcker K., Mocker D., Münze R. and Beyer G.J. (1988)

Stability of lanthanide-protein complexes

Int.J.Appl.Radiat.Isot. 39, 261-264

20. Zak O. and Aisen P. (1988)

Spectroscopic and thermodynamic studies on the binding of gadolinium(III) to human serum transferrin

Biochemistry 27, 1075-1080 
THE INFLUENCE OF EDTMP-CONCENTRATION ON THE BIODISTRIBUTION OF RADIO-LANTHANIDES AND 225-AC IN TUMOR BEARING MICE

G.J.BEYER et al.

\section{Tab. and Fig. CAPTIONS}

Tab.1 Influence of EDTMP ligand concentration on the biodistribution of radio-lanthanides and $225 \mathrm{Ac}$ in tumor bearing nude mice The radionuclides were simultanously injected. The values are expressed as \% injected dose per gram tissue, in case of urine in $\%$ of injected dose and represent averages of 1 to 4 animals, (n.d. = not determined).

Tab.2 Tumor to organ ratios of radionuclide enrichment as function of EDTMP ligand concentration ( .d. = not determined)

Fig.1 Influence of EDTMP ligand concentration of the bio-distribution of radio-lanthanides and $225 \mathrm{Ac}$ in tumor bearing nude mice (in case of urine data are given as $\%$ of injected dose)

Fig.2 Bio-distribution of radio-lanthanides and 225Ac injected in $10 \mathrm{mMol}$ EDTMP solution as function of the ionic radius of the injected radionuclide

(in case of urine data are given as \% of injected dose)

Fig.3 Tumor to liver ratio of radionuclide uptake for radio-lanthanides and $225 \mathrm{Ac}$ as function of the EDTMP ligand concentration

Fig.4 Tumor to organ ratios of radionuclide uptake as function of the ionic radius of the injected radionuclide 


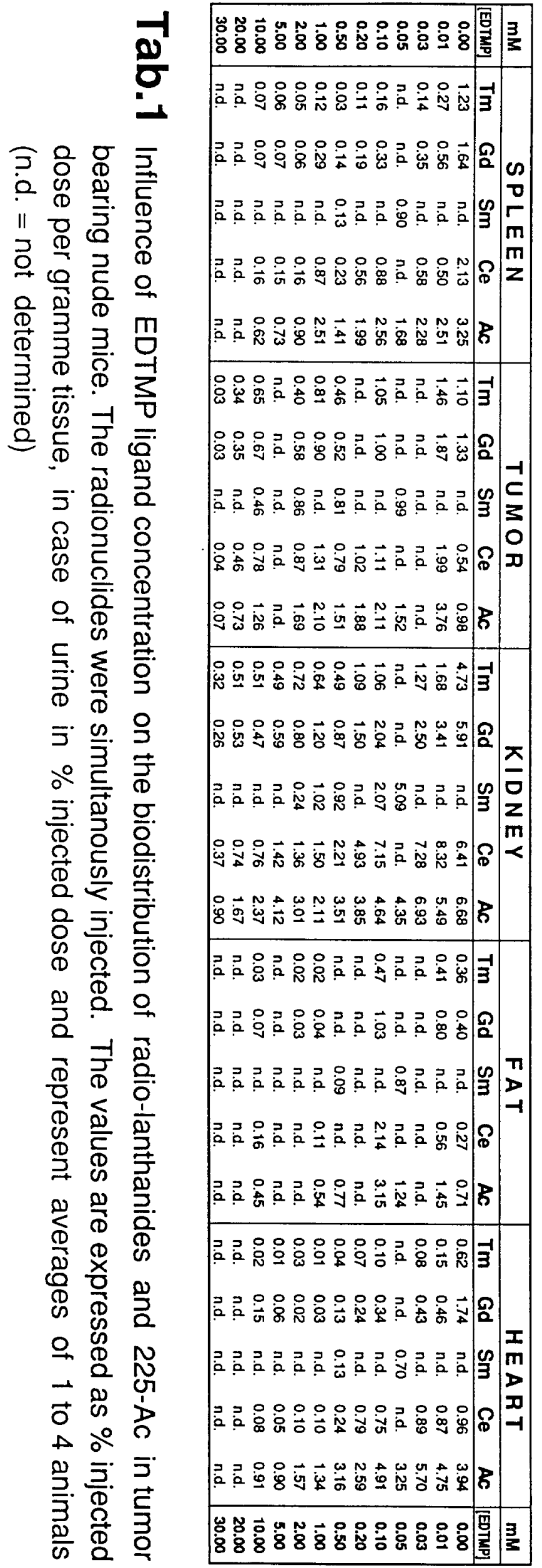

\section{$\overrightarrow{\hat{N}}$}

$\infty$

\section{离}

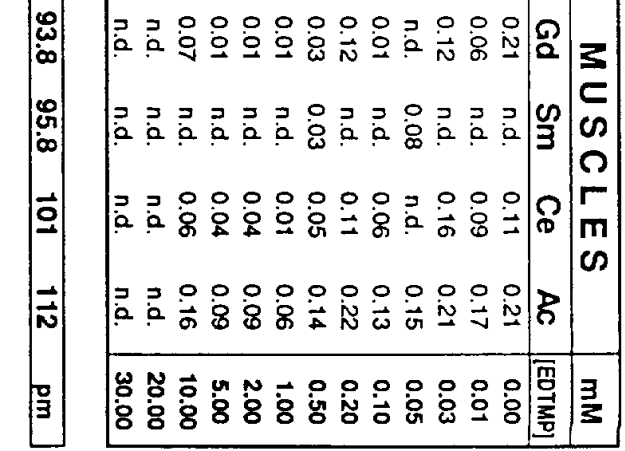

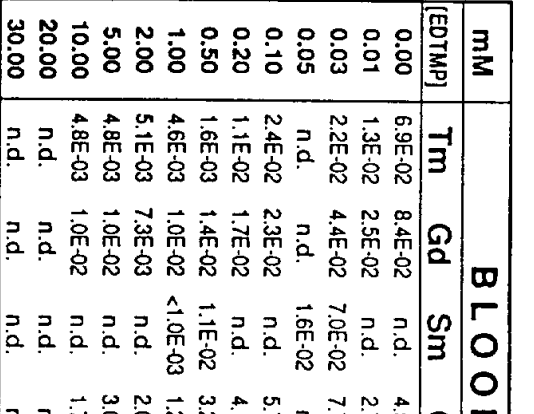

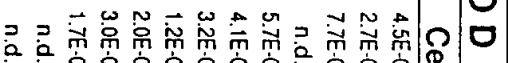

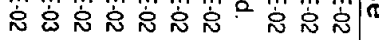

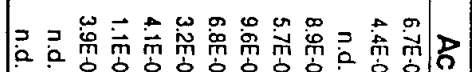

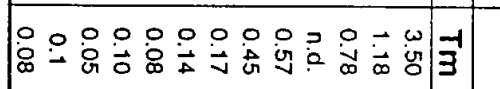

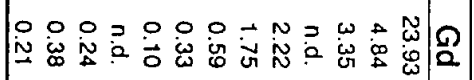

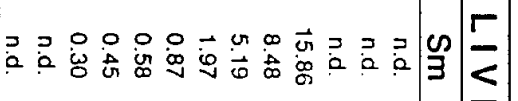

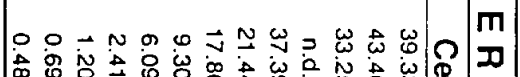

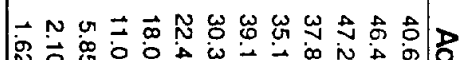

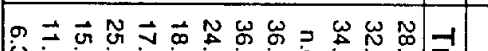

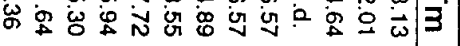

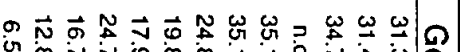

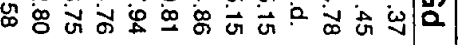

จ

Q

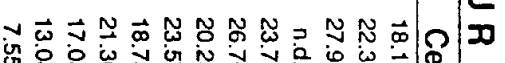

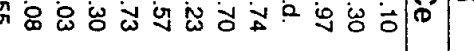

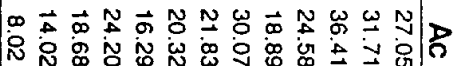

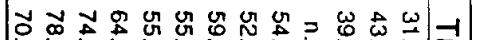

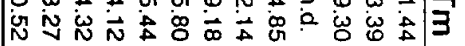

จ

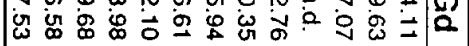

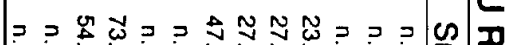

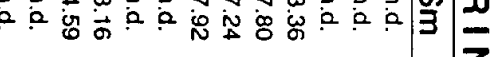

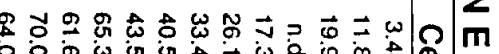

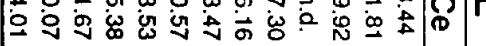

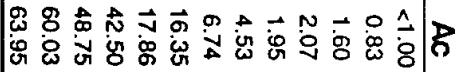

$\Rightarrow=0000000=000-1$

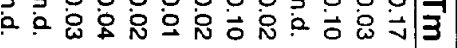
. 


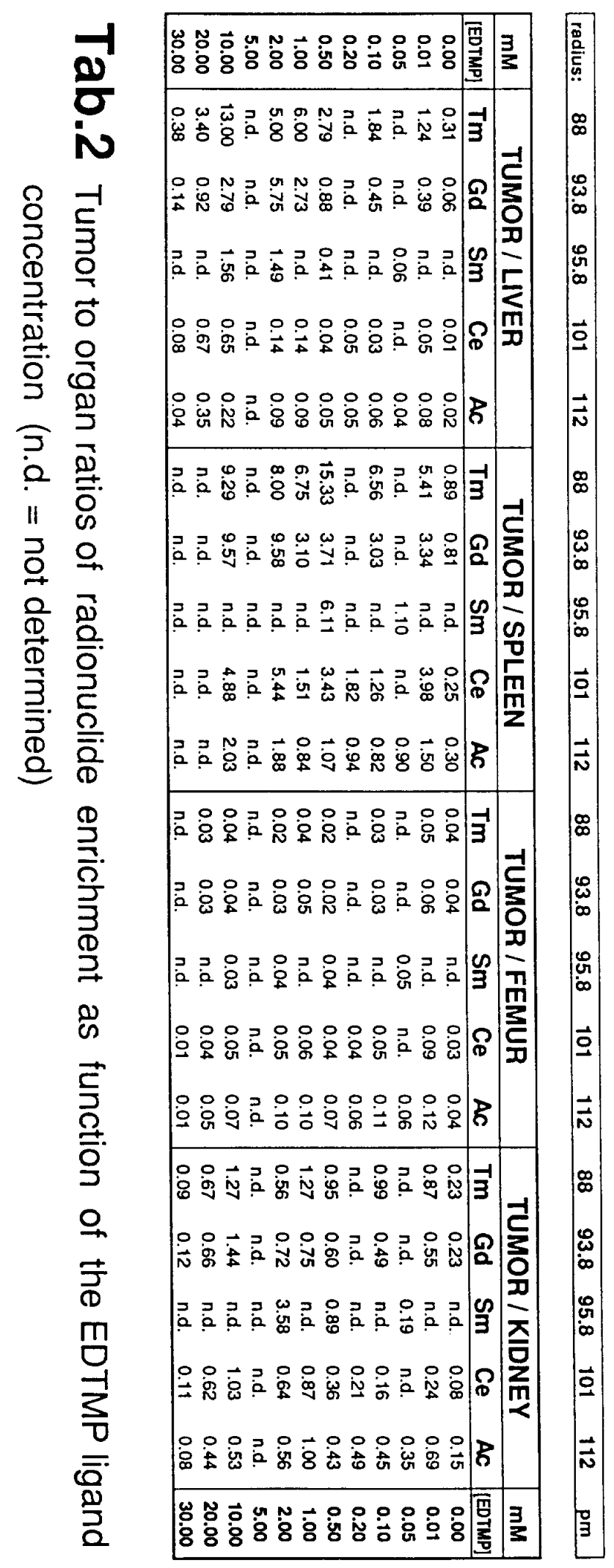

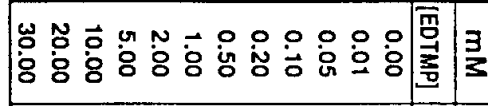

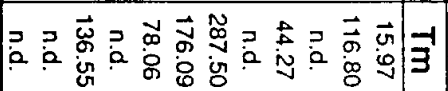

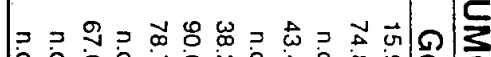

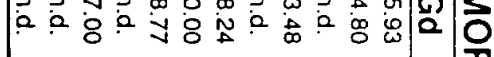

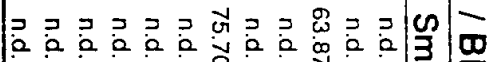

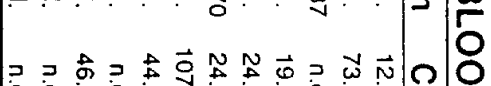

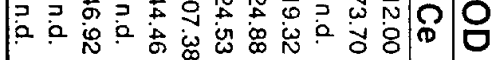

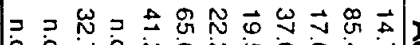
$\because \begin{gathered}0 \\ 0\end{gathered}$

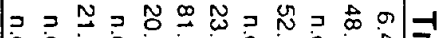

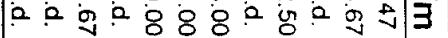

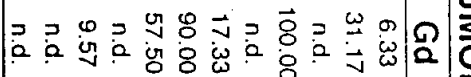

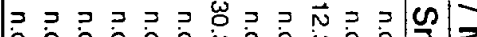

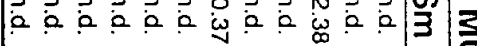

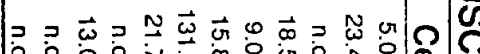
웅

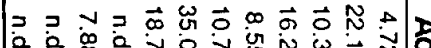
유.

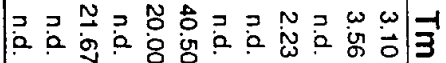

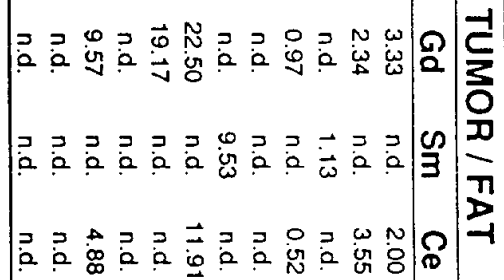

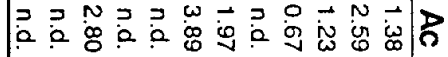

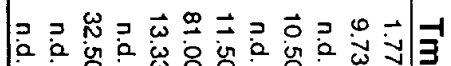

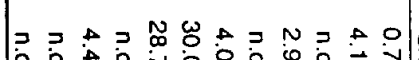

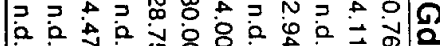

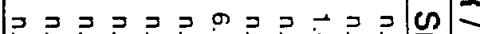

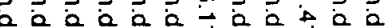

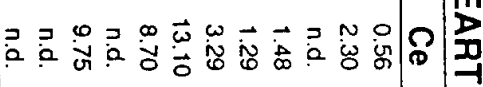

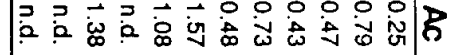

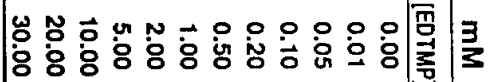




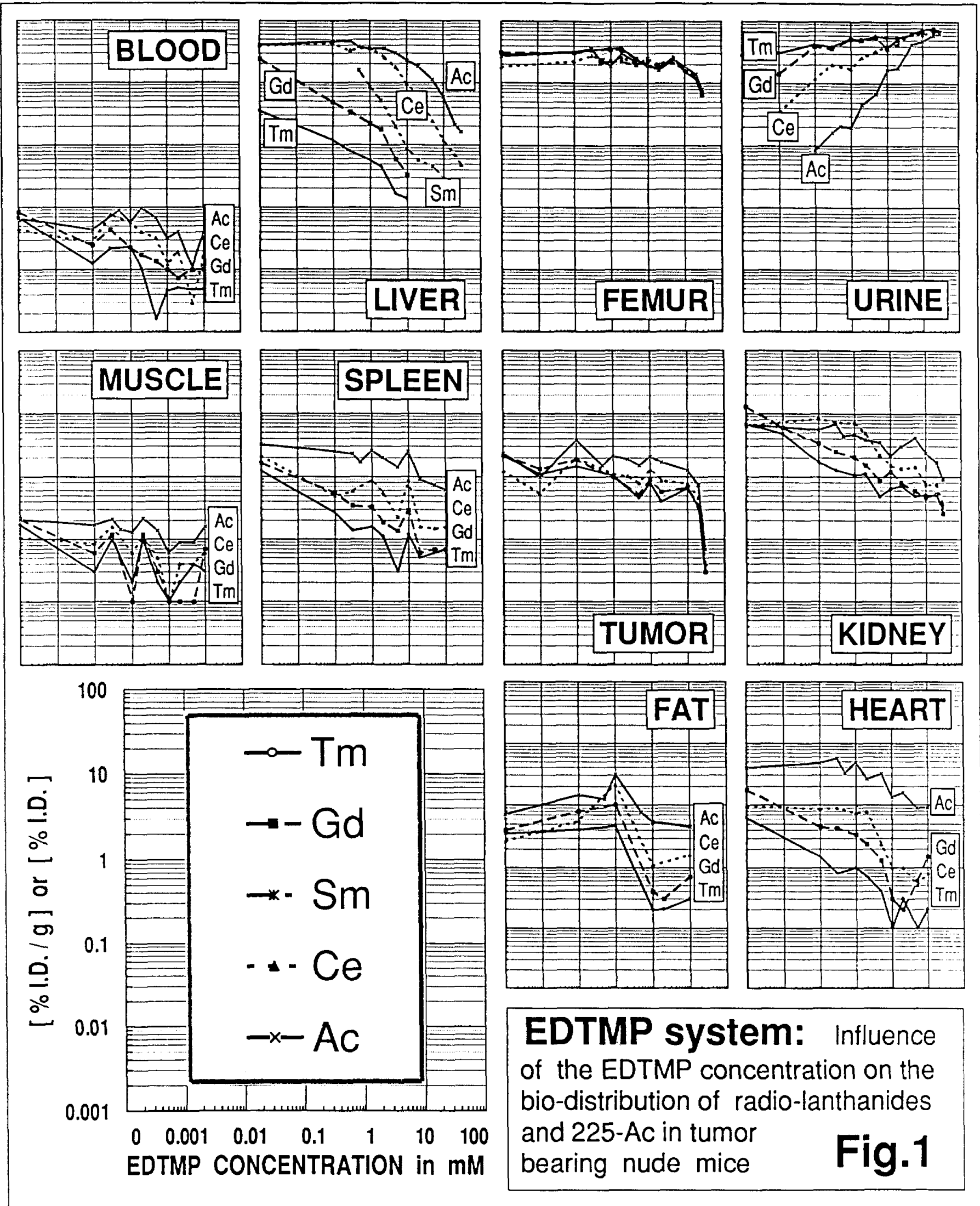




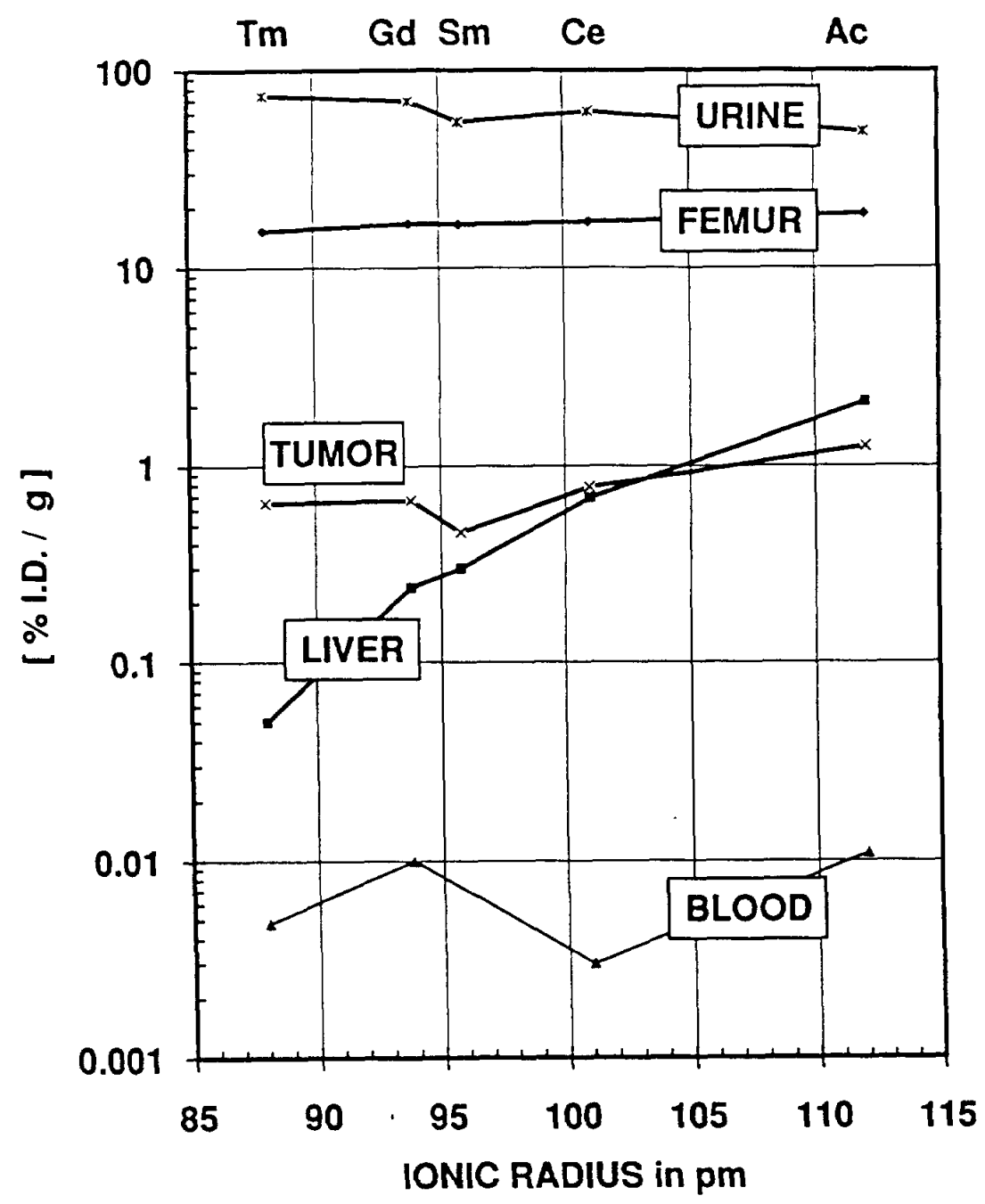

Fig. 2

Bio-distribution of radio-lanthanides and 225 Ac injected in $10 \mathrm{mMol}$ EDTMP solution as function of the ionic radius of the injected radionuclide (in case of urine data are given as \% of injected dose) 


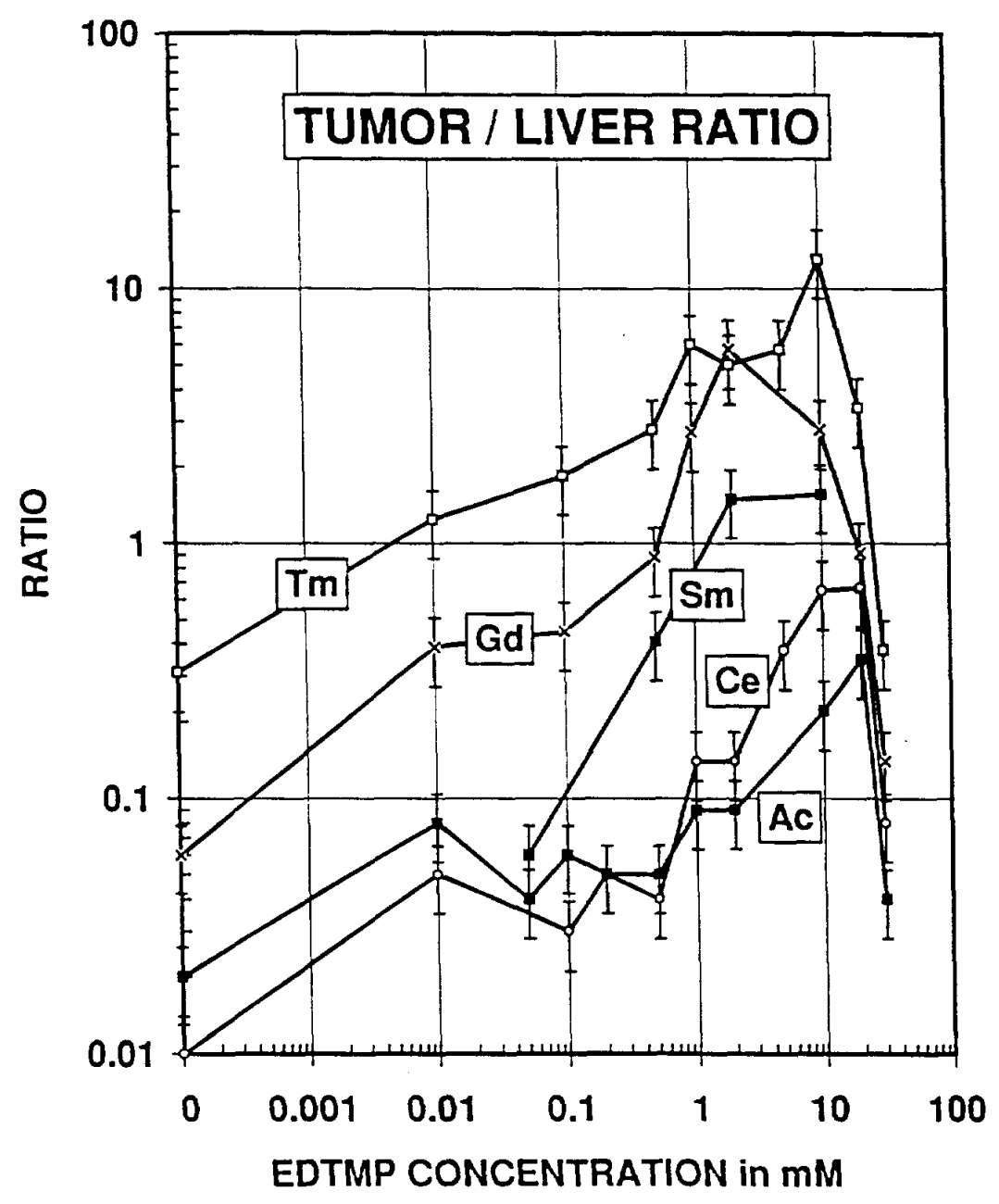

Fig.3

Tumor to liver ratio of radionuclide uptake for radio-lanthanides and $225 \mathrm{Ac}$ as function of the EDTMP ligand concentration 


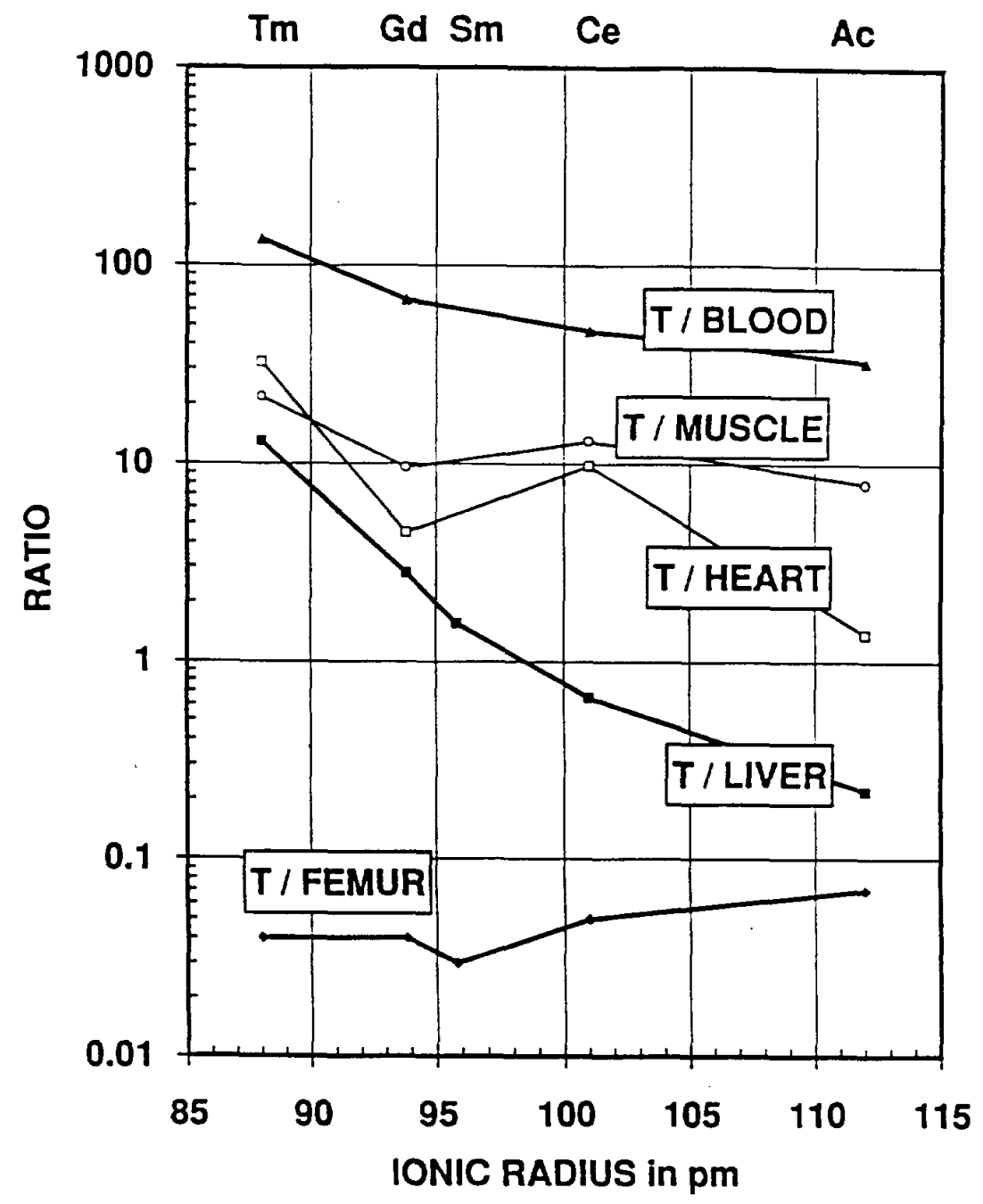

Fig.4

Tumor to organ ratios of radionuclide uptake as function of the ionic radius of the injected radionuclide 